\title{
Initial Conditions for Defining an Arrow of Time at the Start of Inflation?
}

\author{
Andrew Walcott Beckwith \\ Physics Department, College of Physics, Chongqing University, Chongqing, China \\ Email: Rwill9955b@gmail.com, abeckwith@uh.edu
}

How to cite this paper: Beckwith, A.W. (2018) Initial Conditions for Defining an Arrow of Time at the Start of Inflation? Journal of High Energy Physics, Gravitation and Cosmology, 4, 787-795.

https://doi.org/10.4236/jhepgc.2018.44044

Received: March 13, 2018

Accepted: October 28, 2018

Published: October 31, 2018

Copyright $\odot 2018$ by author and Scientific Research Publishing Inc. This work is licensed under the Creative Commons Attribution International License (CC BY 4.0).

http://creativecommons.org/licenses/by/4.0/

\begin{abstract}
This investigation sets forth initial conditions for a start of the arrow of time in cosmology based upon the idea that of having initial degrees of freedom set as $g_{*} \sim 1000$ initially, instead of a maximum value of $g_{*} \sim 100-120$ for the number of degrees of freedom during the electro weak era.
\end{abstract}

\section{Keywords}

Times Arrow, Degrees of Freedom

\section{Introduction}

Recently, Beckwith asked [1] if the following could occur, $S \equiv[E-\mu N] / T \rightarrow S \propto T^{3}$ by setting the chemical potential $\mu \rightarrow 0$ with initial entropy $S \sim 10^{5}$ at the beginning of inflation. Conventional discussions of the arrow of time state that as the Universe grows its temperature drops, which leaves less energy available to perform useful work in the future than that available in the past. Thus the Universe itself has a well-defined thermodynamic arrow of time. The problem of the initial configuration of the arrow of time, however, is not brought up. This paper is to initiate how to set up a well defined initial starting point for the arrow of time. Specifically re-setting the degrees of freedom of about $g_{*} \sim 100-120$ [2] of the electro weak era, to $g_{*} \sim 1000$ at the onset of inflation [1], $S_{\text {initial }} \propto T^{3}$ may be permitted. If the initial temperature of an emerging universe was very low, scaling $S \propto T^{3}$ may be a way to get an arrow of time, with respect to thermal temperatures, alone, with the graviton count a later, emergent particle phenomenon. 


\section{What Can Be Said Initially about Usual Arrow of Time Formulations of Early Cosmology?}

Usual treatments of the arrow of time, i.e. the onset of entropy. The discussion below makes the point that expansion of the universe in itself does not "grow" entropy.

The entropy density $s$ of a radiation field of temperature $T$ is $s \sim T^{3}$. The entropy $S$ in a given comoving volume $V$ is $S=s V$. Since the commoving volume $V$ increases as the universe expands, we have $V \sim R^{3}$. And since the temperature of the microwave background goes down as the universe expands: $T \sim 1 / R$, we have the result that the entropy of a given comoving volume of given space $S \sim R^{-3}$ * $R^{3}=$ constant. Thus the expansion of the universe by itself is not responsible for any entropy increase. There is no heat exchange between different parts of the universe. The expansion is adiabatic and isentropic: $\mathbf{d} \mathbf{S}$ expansion $=0$. i.e. $\mathrm{a}$ process has to be initiated in order to start entropy production.

This discussion above is to emphasize the importance of an initial process for the onset and the growth of entropy. We will initiate candidates for making sense of the following datum.

To measure entropy in cosmology we can count photons. If the number of photons in a given volume of the universe is $N$, then the entropy of that volume is $S \sim k N$ where $k$ is called here Boltzmann's constant.

Note that Y. Jack Ng. has [3], from a very different stand point derived $S \sim n$ based upon string theory derived ideas, with $\boldsymbol{n}$ a "particle" count, which in Y. Jack Ng's procedure is based upon the number of dark matter candidates in a given region of phase space. Y. Jack Ng's idea was partly based upon the idea of quantum "infinite" statistics, and a partition function, details of which will be in Appendix A below.

This counting procedure is different from traditional notions. To paraphrase them, one can state that "The reason why entropy is increasing is because there are stars in that 'box' (unit of phase space used for counting contributions to entropy). Hydrogen fuses to helium and nuclear energy is transformed into heat." i.e. the traditional notion would be akin to heat production due to, initially start BBN nucleosynthesis, and then, frankly, star production/nuclear burning. i.e. one would need to have nuclear processes to initiate heat production. This idea of heat production is actually similar to setting $S \propto T^{3}$, with heat production due to either BBN/ hydrogen burning leading to an increase in temperature, $T$. In this manuscript, we make use of, if $S \equiv[E-\mu N] / T \rightarrow S \propto T^{3}$ by setting the chemical potential $\mu \rightarrow 0$ with initial entropy $S \sim 10^{5}$ at the beginning of inflation. This entails, as we will detail, having increased number of degrees of freedom, initially, with re setting the degrees of freedom of about $g_{*} \sim 100-120$ of the electro weak era, to $g_{*} \sim 1000$ at the onset of inflation, I.e. what will be examined will be the feasibility of the following:

$S \equiv[E-\mu N] / T \longrightarrow \mu \rightarrow 0$ $S \propto T^{3} \approx n$, with $\mathrm{n}$ an initial "quantum unit" count in phase space of Planckian dimensions, where $S \sim 10^{5}$ at the beginning of infla- 
tion. Let us now look at how to initiate such a counting algorithm if one is looking at, say, highly energized gravitons, initially, as part of a counting "algorithm”.

\subsection{Estimating the Size of Contribution to Energy in $S \equiv E / T$,} Assuming a Frequency $v \sim 10^{10}$ Hertz for Relic Gravitons, If the Standard Chemical Potential Is Effectively $\mu=0$ at the Onset of Creation

As suggested earlier by Beckwith [4], gravitons may have contributed to the re-acceleration of the universe one billion years ago. Here, we are making use of refining the following estimates. In what follows, we will have even stricter bounds upon the energy value (as well as the mass) of the graviton based upon the geometry of the quantum bounce, with a radii of the quantum bounce on the order of $l_{\text {Planck }} \sim 10^{-35}$ meters [1] [5].

$$
\begin{aligned}
& \left.m_{\text {graviton }}\right|_{\text {RELATIVISTIC }}<4.4 \times 10^{-22} h^{-1} \mathrm{eV} / \mathrm{c}^{2} \\
& \Leftrightarrow \lambda_{\text {graviton }} \equiv \frac{\hbar}{m_{\text {graviton }} \cdot c}<2.8 \times 10^{-8} \text { meters }
\end{aligned}
$$

For looking at the onset of creation, with a bounce; if we look at $\rho_{\text {max }} \propto 2.07 \cdot \rho_{\text {planck }}$ for the quantum bounce with a value put in for when $\rho_{\text {planck }} \approx 5.1 \times 10^{99}$ grams/ meter ${ }^{3}$, where [1]

$$
E_{\text {eff }} \propto 2.07 \cdot l_{\text {Planck }}^{3} \cdot \rho_{\text {planck }} \sim 5 \times 10^{24} \mathrm{GeV}
$$

Then, taking note of this, one is obtaining having a scaled entropy of $S \equiv E / T \sim 10^{5}$ when one has an initial Planck temperature $T \approx T_{\text {Planck }} \sim 10^{19} \mathrm{GeV}$. One needs, then to consider, if the energy per given graviton is, if a frequency $v \propto 10^{10} \mathrm{~Hz}$ and $E_{\text {graviton-effective }} \propto 2 \cdot h v \approx 5 \times 10^{-5} \mathrm{eV}$, then [1]

$$
S \equiv E_{\text {eff }} / T \sim\left[10^{38} \times E_{\text {graviton-effective }}\left(v \approx 10^{10} \mathrm{~Hz}\right)\right] /\left[T \sim 10^{19} \mathrm{GeV}\right] \approx 10^{5}
$$

Having said that, the $\left[E_{\text {graviton-effective }} \propto 2 \cdot h v \approx 5 \times 10^{-5} \mathrm{eV}\right]$ is $10^{22}$ greater than the rest mass energy of a graviton if $E \sim m_{\text {graviton }}[$ red-shift $\sim 0.55] \sim\left(10^{-27} \mathrm{eV}\right)$ grams is taken when applied to Equation (1.2) above.

\subsection{The Electro Weak Generation Regime of Space Time for Entropy and Early Universe Graviton Production before Eletro-Weak Transitions}

A typical value and relationship between an inflaton potential $V[\phi]$, and a hubble parameter value, $H$ is [1]

$$
H^{2} \sim V[\phi] / m_{\text {Planck }}^{2}
$$

Also, if we look at the temperature $T^{*}$ occurring about the time of the Electro weak transition, if $T \leq T^{*}$ when $T^{*}=T_{c}$ was a critical value, (of which we can write $v\left(T_{c}\right) / T_{c}>1$, where $v\left(T_{c}\right)$ denotes the Higgs vacuum expectation value at the critical temperature $T_{c}$, i.e. $v\left(T_{c}\right) / T_{c}>1$ according to C. Balazc et al. 
(2005) [6] and denotes that the electro weak transition was a "strongly first order phase transition") then one can write, by conventional theory that

$$
H \sim 1.66 \cdot\left[{\sqrt{\tilde{g}_{*}}}\right] \cdot\left[T^{2} / m_{\text {Planck }}^{2}\right]
$$

Here, the factor put in, of $\tilde{g}_{*}$ is the number of degrees of freedom. Kolb and Turner [2] put a ceiling of about $\tilde{g}_{*} \approx 100-120$ in the early universe as of about the electro weak transition. If, however, $\tilde{g}_{*} \sim 1000$ or higher for earlier than that, i.e. up to the onset of inflation for temperatures up to $T \approx T_{\text {Planck }} \sim 10^{19} \mathrm{GeV}$, it may be a way to write, if we also state that $V[\phi] \approx E_{\text {net }}$ that if $[1]$

$$
S \sim 3 \frac{m_{\text {Plank }}^{2}\left[H=1.66 \cdot \sqrt{\tilde{g}_{*}} \cdot T^{2} / m_{\text {planck }}\right]^{2}}{T} \sim 3 \cdot\left[1.66 \cdot \sqrt{\tilde{g}_{*}}\right]^{2} T^{3}
$$

Should the degrees of freedom hold, for temperatures much greater than $T^{*}$, and with $\tilde{g}_{*} \approx 1000$ at the onset of inflation, for temperatures, rising up to, say $T \sim 10^{19} \mathrm{GeV}$, from initially a very low level, pre inflation, then this may be enough to explain how and why certain particle may arise in a nucleated state, without necessarily being transferred from a prior to a present universe.

Furthermore, if one assumes that $S \propto T^{3}$ [5] when $\tilde{g}_{*} \approx 1000$ or even higher even if $T \sim 10^{19} \mathrm{GeV} \gg T^{*}$, then there is the possibility that $S \propto T^{3}$ when $\tilde{g}_{*} \approx 1000$ could also hold, if there was in pre inflationary states very LOW initial temperatures, which rapidly built up in an interval of time, as could be given by $0<t<t_{\text {Planck }} \sim 10^{-44}$ seconds [1].

\subsection{Justification for Setting $\tilde{g}_{*} \approx 1000$ Initially}

H. de La Vega, in conversations with the author in Colmo, Italy, 2009 [7]. flatly ruled out having $\tilde{g}_{*} \approx 1000$ initially. What will be presented here will be a justification for taking this step which $\mathrm{H}$. de La Vega says is not measurable and possible. The author points to, among other things, the Wheeler de Witt derivation for a wave function of the universe, as given by M. Morris [8] (1989) in perturbative super space, with no restriction on the degrees of freedom. While the WdW style of stellar evolution is now out of fashion, something akin to obtaining an initial "wavefunction of the universe" as given in his Equation (3.1) of his article is, by the authors view, necessary, to make sense out of initial conditions appropriate for $S \propto T^{3} \sim n$ when $\tilde{g}_{*} \approx 1000$. The count, $n$, would be in terms of a procedure brought up by both Beckwith, [1] and Mukhanov [9] on page 82 of his book leading to a Bogoluybov particle number density of becoming exponentially large, where $\eta_{1}$ is a time evolution factor, which we can set $\left|\eta_{1}\right| \sim O\left(\beta \cdot t_{\text {Planck }}\right)$, with $\beta$ some numerical multiplicative factor for the Planck interval of time $t_{\text {Planck }}[1][9]$

$$
n \sim \sinh ^{2}\left[m_{0} \eta_{1}\right]
$$

If so, then one can also ask if there is a linkage between the initial conditions, as pertinent to early inflation, and Beckwith's model of re acceleration of the 
universe one billion years ago.

\section{Linkage between the Initial Onset of Inflation, and Reacceleration of the Universe One Billion Years Ago?}

The following is speculative, and if confirmed through additional research would be a major step toward a cosmological linkage between initial inflation, and re acceleration of the universe one billion years ago [2]. Look at A. Yurov's [10] double inflation hypothesis, i.e. Claim: there exist one emergent complex scalar field $\Phi$ and that its evolution in both initial inflation and re acceleration is linked. i.e. he states that this scalar field would account for both 1st and 2nd inflation

Potential in both cases chaotic inflation of the type [10]

$$
V=\vec{m}^{2} \Phi^{*} \Phi
$$

The "mass" term would be, then, as Beckwith understands it, for early universe versions of the Friedman equation

$$
\vec{m} \approx \sqrt{\frac{3}{8}} \cdot\left[\left.\sqrt{\frac{3 H^{2}}{4 \pi G}}\right|_{\text {time } 10^{-35} \mathrm{sec}}+\left.\sqrt{\frac{3 H^{2}}{4 \pi G}}\right|_{\text {time } 10^{-44} \mathrm{sec}}\right]
$$

Furthermore, its bound would be specified by having

$$
|\vec{m}| \leq\left[\frac{l^{2}}{4}\right]
$$

The term, $l$ would be an artifact of five dimensional space time, as provided in a metric as given by Maarten's [11] as

$$
\left.\mathrm{d} S^{2}\right|_{5-\operatorname{dim}}=\frac{l^{2}}{z^{2}} \cdot\left[\eta_{u v} \mathrm{~d} x^{\mu} \mathrm{d} x^{v}+\mathrm{d} z^{2}\right]
$$

The $2^{\text {nd }}$ scalar fields as Yurov [10] writes them contributing to the $2^{\text {nd }}$ inflation, which Beckwith represents [2] is

$$
\varphi_{0,-}=\sqrt{2 / 3} \cdot \vec{m} \cdot\left[t_{1 \mathrm{st}-\mathrm{EXIT}} \sim 10^{-35} \mathrm{sec}\right]
$$

And

$$
\varphi_{+}=\left[\varphi_{0,+}^{3}-\sqrt{3 / 2} \cdot \frac{3 M^{2} t}{\vec{m}}\right]^{1 / 3}
$$

As Beckwith sees it, making a full linkage between Yurov's formalism [10] for double inflation, Beckwith's re acceleration graphics [2], and initial inflationary dynamics, as referenced by obtaining $n_{f} \approx 10^{6}$ to $10^{7}$ would be to make the following relations between Yurov's [10] versions of the Friedman equations, and what Beckwith [2] did,

$$
H^{2}=\frac{1}{6} \cdot\left[\dot{\varphi}^{2}+\vec{m}^{2} \varphi^{2}+\frac{M^{2}}{\varphi^{2}}\right] \leftrightarrow\left(\frac{\tilde{\kappa}^{2}}{3}\left[\rho+\frac{\rho^{2}}{2 \lambda}\right]\right)+\frac{m}{a^{4}}
$$

As well as having: 


$$
\dot{H}=V-3 H \leftrightarrow \dot{H} \cong \frac{2 m}{a^{4}}
$$

The left hand side of both Equation (1.14) and Equation (1.15) are Yurov's [10], and the right hand side of both Equation (1.14) and Equation (1.15) above are Beckwith's adaptation [1] of modification of Maarten's brane theory [11] work which was used in part to obtain the re acceleration of the universe graphics Beckwith obtained [2] a, i.e. the behavior of massive gravitons one billion years ago to mimic DE in terms of the re acceleration parameter IN any case, the following would be needed to be verified to make the linkage.

$$
\left.\frac{3 H^{2}}{4 \pi G} \gg V(t)\right|_{\text {time }-10^{-44} \text { sec }}
$$

i.e. that the potential energy, $V$, of initial inflation is initially over shadowed by the contributions of the Friedman equation, $H$, at the onset of inflation.

We should note, that the potential energy as stated would be assuming that Equation (1.16) has consistency with Equation (1.5), for very large temperatures.

\section{Conclusions}

A way to obtain traces of information exchange, from prior to present universe cycles is finding a linkage between information and entropy. If such a parameterization can be found and analyzed, then Seth Lloyd's [12] shorthand for entropy,

$$
I=S_{\text {total }} / k_{B} \ln 2=[\# \text { operations }]^{3 / 4}=\left[\rho \cdot c^{5} \cdot t^{4} / \hbar\right]^{3 / 4}
$$

could be utilized as a way to represent information which can be transferred from a prior to the present universe. The question is to ask, if Equation (1.17) permits a linkage of gravitons as information carriers, can there be a linkage of information, in terms of the appearance of gravitons in the time interval of, say $0<t<t_{\text {Planck }}$ either by vacuum nucleation of gravitons/information packets. Appropriate values/inputs into $\rho$ are being considered along the lines of graviton mass/contributions along the lines brought up in this paper already.

An alternative to Equation (1.7) if one sees no way of implementing what Ng. suggested via his infinite quantum statistics [3] would be to look at thermal inputs from a prior to the present universe, as suggested by L. Glinka [13] [14]

$$
n_{f}=[1 / 4] \cdot\left[\sqrt{\frac{v\left(a_{\text {initial }}\right)}{v(a)}}-\sqrt{\frac{v(a)}{v\left(a_{\text {final }}\right)}}\right]
$$

As well as, if $h_{0} \sim 0.75$

$$
\Omega_{g w}(v) \cong \frac{3.6}{h_{0}^{2}} \cdot\left[\frac{n_{f}}{10^{37}}\right] \cdot\left(\frac{v}{1 \mathrm{kHz}}\right)^{4}
$$

If we take into consideration having $a \sim a_{\text {final }}$, then Equation (1.14) above will, in most cases be approximately 


$$
n_{f}=[1 / 4] \cdot\left[\sqrt{\frac{v\left(a_{\text {initial }}\right)}{v(a)}}-1\right] \sim[1 / 4] \cdot\left[\sqrt{\frac{v\left(a_{\text {initial }}\right)}{v(a)}}\right]
$$

For looking at $\Omega_{g} \approx 10^{-5}-10^{-14}$, with $\Omega_{g} \approx 10^{-5}$ in pre-big bang scenarios, with initial values of frequency set for $v\left(a_{\text {initial }}\right) \approx 10^{8}-10^{10} \mathrm{~Hz}$, as specified by Grishkuk [15] $v\left(a_{\text {final }}\right) \approx 10^{0}-10^{2} \mathrm{~Hz}$ near the present era, and $a \sim\left[a_{\text {final }}=1\right]-\delta^{+}$, i.e. close to the final value of today's scale value, Filling in/choosing between either implementation of Equation (1.7), or Equation (1.10) will be what the author is attempting to do in the foreseeable future. i.e. if one can use [3]

$$
S \approx n
$$

using Equation (1.10) directly, or if $S \neq n$, using Equation (1.10), but instead uses $S \propto T^{3}$, with temperature rapidly increasing from a low value to $T_{\text {Planck }} \approx 10^{19} \mathrm{GeV}$ in about a time interval during the onset of inflation, for the beginning of the arrow of time, in cosmology. Beckwith views determining if the degrees of freedom initially could go as high as $\tilde{g}_{*} \approx 1000$ or even higher even if $T \sim 10^{19} \mathrm{GeV}$ as essential in determining the role of $S \propto T^{3}$, as temperatures go from an initial low point, to $T \sim 10^{19} \mathrm{GeV}$ for understanding the role of thermal heat transfer in the arrow of time issue.

Note, very importantly, any coupling between Equation (1.5) and Equation (1.16) probably requires that there is no initially low temperature behavior, pre-inflation, prior to the rise of temperature of the Quantum Planck Temperature of $\sim 10^{19} \mathrm{GeV}$.

\section{Open Question: Do We Have a Match Up with Smoot's Ercole Challonge Table? Guess as to Possible Outcomes Presented}

In a colloquium presentation done by Dr. Smoot in Paris [16] (2007); he alluded to the following information theory constructions which bear consideration as to how much is transferred between a prior to the present universe in terms of information "bits".

1) Physically observable bits of information possibly in present Universe- $10^{180}$

2) Holographic principle allowed states in the evolution/development of the Universe- $10^{120}$

3) Initially available states given to us to work with at the onset of the inflationary era $-10^{10}$

4) Observable bits of information present due to quantum/statistical fluctuations $-10^{8}$

Our guess is as follows. That the thermal flux from a prior to the present universe may account for up to $10^{10}$ bits of information. These could be transferred from a prior universe to our present, and that there could be, perhaps $10^{120} \mathrm{mi}-$ nus $10^{10}$ bytes of information temporarily suppressed during the initial changing of fermion states of matter to a bosonic phase of matter right at the onset of the big bang itself. 
Then after the degrees of freedom dramatically drops during the beginning of the descent of temperature from about $T \approx 10^{32}$ Kelvin to at least three orders of magnitude less, as we move out from an initial red shift

$$
z \approx 10^{25}
$$

To [17]

$$
T \approx \sqrt{\varepsilon_{V}} \times 10^{28} \text { Kelvin } \sim T_{\text {Hawkings }} \cong \frac{\hbar \cdot H_{\text {initial }}}{2 \pi \cdot k_{B}}
$$

Whichever model we can come up with that does this is the one we need to follow, experimentally. And it gives us hope in confirming if or not we can eventually analyze the growth of structure in the initial phases of quantum nucleation of emergent space time [18]. In the end, in future research, we will delineate how this idea which is offered above, ties in with cosmological expansion. In particular, we wish to investigate what is brought up in [19] which we do not believe is correct. We don't believe that the cosmological constant is negative. We will in future research use this above analysis as to critique what is done in [19] with an eye toward falsifying it. We also will tie in our treatment of this topic with a tie into the work provided in [20] which was presented as a contribution to the Frontiers in Fundamental physics 15 conference. i.e. attempt not only to falsify [19] but to show its relevance to [20].

\section{Acknowledgements}

This work is supported in part by National Nature Science Foundation of China grant No. 11375279.

\section{Conflicts of Interest}

The authors declare no conflicts of interest regarding the publication of this paper.

\section{References}

[1] Beckwith, A.W. (2010) Could Gravitons from Prior Universe Survive Quantum Bounce to Reappear in Present Universe. http://vixra.org/abs/1008.0050

[2] Kolb, E. and Turner, S. (1994) The Early Universe. Westview Press, Chicago.

[3] Ng, Y.J. (2008) Spacetime Foam: From Entropy and Holography to Infinite Statistics and Nonlocality. Entropy, 10, 441-461. https://doi.org/10.3390/e10040441

[4] Beckwith, A.W. (2010) Entropy Growth in the Early Universe, and the Search for Determining if Gravity is Classical or Quantum Foundations (Is Gravity a Classical or Quantum Phenomenon at Its Genesis 13.7 Billion Years Ago?) http://vixra.org/abs/0910.0057

[5] Valev, D. (2008) Neutrino and Graviton Rest Mass Estimations by a Phenomenological Approach. Aerospace Research in Bulgaria, 22, 68-82. http://arxiv.org/abs/hep-ph/0507255

[6] Balazs, C., Carena, M., Menon, A., Morrissey, D.E. and Wagner, C.E.M. (2005) Overview of Electroweak Baryogenesis. 2005 ALCPG \& ILC Workshops, Snowmass. http://www.slac.stanford.edu/econf/C0508141/proc/papers/ALCPG0333.PDF 
[7] de Vega, H. (2009) Inflation in the Standard Model of the Universe. http://isapp2009.mib.infn.it/media/lectures/devega.pdf

[8] Morris, M.S. (1989) Initial Condition for Perturbations in $R+\varepsilon R^{2}$ Cosmology. Physical Review D, 39, 1511-1516.

http://authors.library.caltech.edu/6670/1/MORprd89.pdf. https://doi.org/10.1103/PhysRevD.39.1511

[9] Mukhanov, V. and Winitzki, S. (2007) Introduction to Quantum Effects in Gravity. Cambridge University Press, Cambridge. https://doi.org/10.1017/CBO9780511809149

[10] Yurov, A. (2002) Complex Field as Inflaton and Quintessence. https://arxiv.org/abs/hep-th/0208129

[11] Maartens, R. (2004) Brane-World Gravity. Living Reviews in Relativity, 7, 7. http://www.livingreviews.org/lrr-2004-7

[12] Lloyd, S. (2002) Computational Capacity of the Universe. Physical Review Letters, 88, Article ID: 237901. https://doi.org/10.1103/PhysRevLett.88.237901

[13] Glinka, L. (2007) Quantum Information from Graviton-Matter Gas. SIGMA, 3, 87-100.

[14] Glinka, L. (2009) New Approach to Quantization of Cosmological Models. Gravitation and Cosmology, 15, 317-322.

[15] Grishchuk, L.P. (2001) Relic Gravitational Waves and Their Detection. Lecture Notes in Physics, 562, 167-194.

[16] Smoot, G. (2007) CMB Observations and the Standard Model of the Universe. https://chalongedevega.fr/Paris07_Smoot.pdf

[17] de Vega, H., Falvella, M. and Sanchez, N. (2009) HIGHLIGHTS and CONCLUSIONS of the Chalonge 13th Paris Cosmology Colloquium: The Standard Model of the Universe: From Inflation to Today Dark Energy.

[18] Ruutu, V., Eltsov, V., Gill, A., Kibble, T., Krusius, M., Makhlin, Y.G., Placais, B., Volvik, G. and Wen, Z. (1996) Vortex Formation in Neutron-Irradiated ${ }^{3} \mathrm{He}$ as an Analog of Cosmological Defect Formation. Nature, 382, 334-336. https://doi.org/10.1038/382334a

[19] Mersini-Houghton, L. (2006) The Arrow of Time Forbids a Positive Cosmological Constant $\Lambda$. https://arxiv.org/abs/gr-qc/0609006

[20] Beckwith, A. (2018) Using "Enhanced Quantization" to Bound the Cosmological Constant, (For a Bound-On Graviton Mass), by Comparing Two Action Integrals (One Being from General Relativity) at the Start of Inflation. http://vixra.org/abs/1802.0305 